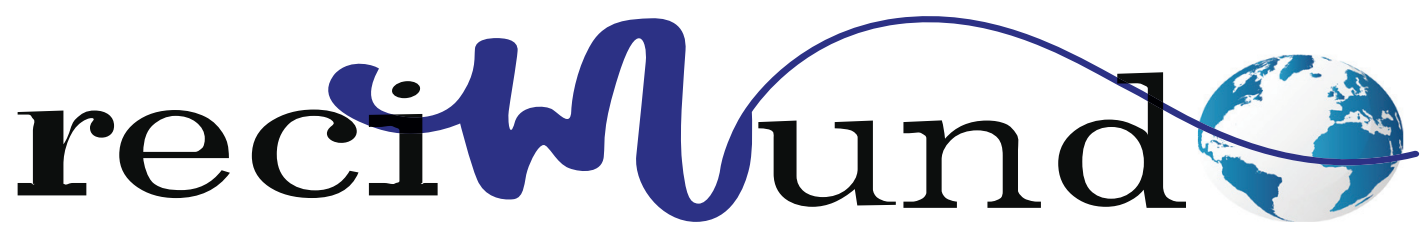

Revista Científica Mundo de la Investigación y el Conocimiento

DOI: 10.26820/recimundo/4.(1).esp.marzo.2020.155-163

URL: http://recimundo.com/index.php/es/article/view/788

EDITORIAL: Saberes del Conocimiento

REVISTA: RECIMUNDO

ISSN: 2588-073X

TIPO DE INVESTIGACIÓN: Artículo de Revisión

CóDIGO UNESCO: 3205 Medicina Interna

PAGINAS: 155-163

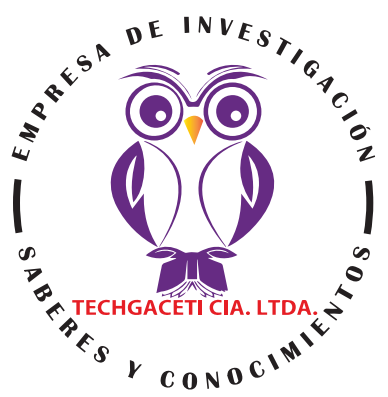

\title{
Uso de anticonceptivos orales en la dismenorrea
}

\section{Use of oral contraceptives in dysmenorrhea} Uso de contraceptivos orais na dismenorreia

\section{Ana Karina Díaz Chávez'; Janyna Nathaly Jaramillo Moreno; Aracely Vanessa Aguilar Cobo; Alexis Javier Haro Perdomo ${ }^{4}$}

\section{RECIBIDO: 20/11/2019 ACEPTADO: 29/01/2020 PUBLICADO: 05/03/2020}

1. Médico; Investigadora Independiente; Quito, Ecuador; akdiazchavez@gmail.com; (D) https://orcid.org/00000003-3328-2377

2. Médico; Investigadora Independiente; Quito, Ecuador; jany_nj@hotmail.com; (D) https://orcid.org/0000-00027203-5435

3. Médico; Investigador Independiente; Quito, Ecuador; aracelyv19@hotmail.com; (DD https://orcid.org/00000002-9489-8012

4. Médico; Investigador Independiente; Quito, Ecuador; javicoalexis@gmail.com; (DD https://orcid.org/0000-00027121-7309

\section{CORRESPONDENCIA \\ Ana Karina Díaz Chávez \\ akdiazchavez@gmail.com \\ Quito, Ecuador}




\title{
RESUMEN
}

Se realizó una búsqueda de la literatura disponible con la finalidad de analizar los efectos producidos por el uso de anticonceptivos orales en la dismenorrea, una de las dolencias relacionadas a la menstruación más importantes. Esta investigación fue del tipo documental enmarcada en una metodología de revisión en la que se determinó primeramente las causas que originan los distintos tipos de dismenorrea, seguido de la influencia que tiene la ingesta de anticonceptivos orales en el tratamiento de la misma y los diversos estudios donde se analiza su eficacia como tratamiento de esta patología. Se concluyó que a pesar de que las mujeres que utilizaron anticonceptivos orales presenciaron reducción en la intensidad de los dolores, no existe certeza en cuanto a la eficacia de este tratamiento, por lo tanto, se deben explorar otras alternativas en caso de que fracase este método.

Palabras clave: Dismenorrea, Prostaglandinas, Anticonceptivos Orales, Estrógeno.

\begin{abstract}
A search of the available literature was carried out in order to analyze the effects produced by the use of oral contraceptives in dysmenorrhea, one of the most important conditions related to menstruation. This research was of the documentary type framed in a review methodology in which the causes of the different types of dysmenorrhea were first determined, followed by the influence of oral contraceptive intake in the treatment of it and the various studies where Its effectiveness is analyzed as a treatment for this pathology. It was concluded that although women who used oral contraceptives witnessed a reduction in pain intensity, there is no certainty as to the efficacy of this treatment, therefore, other alternatives should be explored in case this method fails.
\end{abstract}

Keywords: Dysmenorrhea, Prostaglandins, Oral Contraceptives, Estrogen.

\section{RESUMO}

Foi realizada uma pesquisa na literatura disponível para analisar os efeitos produzidos pelo uso de contraceptivos orais na dismenorreia, uma das condições mais importantes relacionadas à menstruação. Esta pesquisa foi do tipo documentário enquadrada em uma metodologia de revisão na qual foram determinadas as causas que originam os diferentes tipos de dismenorreia, seguidas pela influência que a ingestão de contraceptivos orais exerce sobre o tratamento e os diversos estudos em que A sua eficácia é analisada como um tratamento para esta patologia. Concluiu-se que, embora as mulheres que usavam contraceptivos orais tenham observado uma redução na intensidade da dor, não há certeza quanto à eficácia desse tratamento, portanto, outras alternativas devem ser exploradas caso esse método falhe.

Palavras-chave: Dismenorreia, Prostaglandinas, Contraceptivos orais, Estrogênio. 


\section{Introducción}

El uso de anticonceptivos como método de prevención de embarazos ha sido ampliamente extendido durante décadas y sus tipos han variado enormemente, desde medicamentos hasta dispositivos, lo que permite elegir el más adecuada y accesible para cada caso, además algunos ayudan a evitar enfermedades de transmisión sexual mientras que otros son poco invasivos y casi ni alteran el estilo de vida de quien los usa. (Oficina para la Salud de la Mujer, 2017)

En el caso de los anticonceptivos orales, constituyen uno de los métodos más populares, debido a que no afecta la actividad sexual y al ser usados de forma apropiada poseen una alta efectividad. Referente a estos, Casey (2018) indica que:

Imitan a las hormonas ováricas una vez ingeridos, inhiben la liberación de la hormona liberadora de gonadotropina $(\mathrm{GnRH})$ por el hipotálamo, inhibiendo así la liberación de las hormonas hipofisarias que estimulan la ovulación. Los ACO también afectan el revestimiento del útero y hacen que el moco cervical se espese, haciéndolo impermeable a los espermatozoides. (pp. 1)

El autor anterior añade que, los beneficios que trae dicho método anticonceptivo son importantes, ya que se les atribuye las propiedades de reducción de riesgo de los canceres de ovario y endometrio, así como diversos trastornos como sangrado anormal, osteoporosis, quistes ováricos y trastornos benignos de la mama.

Asimismo, otro estudio señala que los anticonceptivos orales pueden ser utilizados para controlar la dismenorrea. Esta problemática se caracteriza por la aparición de "dolor pélvico crónico de origen ginecológico que se presenta durante el periodo menstrual, siendo también descrito como calambres o cólicos menstruales o menstruación dolorosa". La misma interfiere de forma negativa a la mujer, limitando la realización de sus actividades cotidianas, alterando su entorno propio, social y profesional, así como también su sexualidad, la cual es altamente afectada. Debido a que esta responde de manera favorable a la inhibición de la ovulación, lo que es un efecto de la ingesta de anticonceptivos orales, que además reducen el fluido menstrual, así como la cantidad de prostaglandinas producidas que en conjunto alivian los dolores causados por la dismenorrea, son altamente promovidos como tratamiento eficaz contra esta problemática. (Amaya, 2010, pág. 1; Wong, Farquhar, Roberts \& Proctor, 2009)

La dismenorrea constituye uno de los motivos más importantes de abandono, tanto escolar como profesional, como lo indican Escalonilla, Rodríguez, Marroyo \& Lillo (2010), quienes la exhiben como uno de las problematicas mas significativas sufridas por las mujeres, debido a su alta incidencia, la cual se encuentra entre los $25 \%$ y $90 \%$ según diversos estudios.

Por otro lado, En un estudio enfocado a discutir las alteraciones menstruales habituales, se expone que es conveniente tomarlas en cuenta debido a que pueden ser la manifestación de una enfermedad crónica o aguda importante. (Schulin \& Conejero, 2011)

Entre estos sufrimientos, uno de los más frecuentes es la dismenorrea, el cual posee mayor prevalencia en adolescentes y adultos en las edades de 20 y 25 años, mientras que, a partir de los 30 años de edad, disminuye la cantidad de casos posibles. (Yañez, Bautista, Ruiz \& Ruiz, 2010)

Al mismo tiempo, Sandoval, Madrid, Sandoval \& Paz (2013) exponen que debido a que cualquier mujer puede padecer de dismenorrea y las probabilidades de ello son altas, esta puede considerarse un problema que afecta a todas las personas que forman parte del ambito social que rodea a las mujeres, por lo tanto debe prestarsele la atencion adecuada. 
Cabe señalar que la dismenorrea se clasifica en primaria y secundaria, como lo indican los autores anteriores, quienes explican que el tipo dependera de la falta o presencia de irregularidades ginecológicas y la espontaneidad del caso.

Por otro lado, Ortiz et al. (2007) mencionan que existe evidencia que apunta que la dismenorrea es ocasionada por "la liberación de prostaglandinas en el fluido menstrual" (pág. 25) lo que produce dolor y contracciones uterinas, por lo cual señalan que los fármacos anti-inflamatorios no esteroideos constituyen también una opción para controlar esta problemática.

A pesar de que los anticonceptivos orales han sido altamente promovidos como los principales aliviadores de la dismenorrea, pocos estudios han sido llevados a cabo para confirmar su eficacia, así como sus efectos adversos, además, estos por lo general poseen metodologías de baja calidad lo que los expone a fallos. (Wong et at., 2009)

En este orden de ideas, Yañez et al. (2010), respecto a la dismenorrea, señalan que "a pesar del conocimiento actual de la fisiopatología y de la amplia gama de opciones terapéuticas para controlar el dolor, sigue siendo una patología con alto impacto en la calidad de vida de mujeres jóvenes" (pág. 39).

Debido a esto, el presente estudio tiene como objetivo analizar uno de los tratamientos mas recomendados para la dismenorrea, como lo es la ingesta de anticonceptivos orales, para determinar asi los factores que influyen en el éxito del tratamiento, considerando tambien los posibles efectos adversos y recomendaciones a tener en cuenta durante el manejo de esta dolencia.

\section{Materiales y Métodos}

A inicio de febrero de 2020, se llevó a cabo una búsqueda estructurada de la literatura encontrada en las bases de datos Science-
Direct, SciELO, PubMed, Medline Plus, Cochrane Library entre otros, con los términos "Dismenorrea", "Tratamiento de la dismenorrea", "Características de la dismenorrea" y "Anticonceptivos orales para tratar la dismenorrea", tanto en inglés como en español. Con el fin de determinar el impacto que tienen los anticonceptivos orales en el manejo de la dismenorrea, se incluyeron revisiones sistemáticas con o sin metaanálisis, artículos científicos, estudio de cohorte, ebooks, y demás documentos que a sensatez de los autores se encontraron recubiertos de las particularidades propias de las investigaciones cientificoacademicas, publicados los últimos 20 años. Se desestimaron casos y controles; series y reportes de casos; artículos de revisión narrativa; editoriales, y cartas al editor.

Como suplemento, se realizó una exploración sin limitación cronológica para descubrir información importante, diferente a lo expuesto en los términos de búsqueda, pero necesaria para el desarrollo de la presente revisión. La inclusión o exclusión de cada una de las referencias en la presente revisión se definió por el consenso de los autores.

\section{Resultados}

Jiménez, González, Mesa \& Capera (2013) expresan que "la dismenorrea es uno de los cuadros cíclicos más comunes dentro de los procesos dolorosos pélvicos crónicos" (pág. 21). Los mismos añaden que existen una prevalencia promedio de 50\% de esta patología.

Los dolores de cabeza, cólicos y náuseas, así como molestias abdominales son otras de las dolencias ocasionadas durante los episodios de dismenorrea. La Organización Mundial de la Salud (OMS) expresó en 1992 que esta problemática se clasifica en primaria y secundaria. Se entiende por dismenorrea primaria a los casos donde no se presencia una patología pélvica relacionada al trastorno. Es ocasionada por "contracciones del miometrio inducido por 
prostaglandinas (principalmente PGF2 $\alpha$ ) producidas y liberadas por el endometrio secretor, las cuales producen contracción miometral importante, vasoconstricción de vasos endometriales y miometriales, isquemia del útero y sensibilización de las terminales nerviosas que en conjunto se va a traducir en dolor". Por otra parte, en cuanto a la dismenorrea secundaria está relacionada a "procesos patológicos como endometriosis, adenomiosis, enfermedad pélvica inflamatoria, tumoraciones, estenosis cervical, malformaciones uterinas, congestión pélvica y dispositivo intrauterino". (Ortiz et at., 2007, págs. 24-25)

Mientras que, entre los factores de riesgo relacionados a la presencia de dismenorrea se encuentran "antecedentes maternos de dismenorrea, realización de ejercicio, consumo de cigarrillo, consumo de café, edad de la menarquia, sangrado durante la menstruación, regularidad de la menstruación, consumo de anticonceptivos y gestaciones previas". (Jiménez et al., 2013, pág. 25)

Como se mencionó anteriormente, las prostaglandinas juegan un papel fundamental en la aparición de dismenorrea primaria, a lo que Muñoz, Camacho, Alvarado \& Macías (2018) señalan que:

Esto es debido a la caída de la progesterona que induce el desprendimiento del revestimiento endometrial para prepararse para la menstruación y, a través de este, el aumento de la producción de prostaglandina. La prostaglandina es una hormona, que se puede saber a partir de la obstetricia como el misoprostol. Los síntomas adicionales de dismenorrea como náuseas y diarrea son los mismos que los efectos secundarios de misoprostol. (pág. 777)

Los autores anteriores añaden que, la producción de prostaglandina aumenta cuando los ciclos menstruales presentan ovulación, debido a ello es habitual que durante los casos dismenorrea primaria comiencen a partir de uno ciclos luego de la menarquia. Por ultimo expresan que no es común que la dismenorrea primaria sea causada por "malformaciones congénitas o adquiridas del tracto reproductor femenino, tales como el himen imperforado, y la obstrucción después de la mutilación genital femenina". (pág. 777)

Por lo cual, Ortiz et al. (2007) señalan la importancia de que se busque el tratamiento adecuado, pero revelan el hecho de que solamente un $0.4 \%$ a un $15.5 \%$ de las mujeres que sufren de dismenorrea acuden a la consulta, mientras que el resto optan por auto medicarse, principal forma de tratamiento en un porcentaje elevado de la población. Entre los medicamentos más usados se encuentran aquellos de venta libre como el "paracetamol, pamabrom y pirilamina (Syncol $($; 44\%), metamizol con butilhioscina (Buscapina $\left.{ }^{\circledR} ; 22.9 \%\right)$, naproxeno (10.1\%), ibuprofeno $(7.3 \%)$ y paracetamol (6.4\%)" (pág. 27), como lo indica el estudio llevado a cabo por los autores anteriores, donde se aplicó un cuestionario a 230 estudiantes entre las edades de 17 a 32 años.

Por otra parte, Wong et al. (2009) exponen que uno de los medicamentos a los que se le atribuyen las propiedades de regular la dismenorrea son los anticonceptivos orales, los cuales han sido utilizados para este propósitos desde su liberación para uso general en 1960. Sin embargo, explican que este tipo de terapia hormonal o endocrina solo es considerada por algunos como potencialmente útil si se desea la anticoncepción a largo plazo. Además, su uso en el tratamiento de la dismenorrea secundaria ha sido cuestionada también, debido a que, a pesar de sus efectos favorables contra esta patología, la causa orgánica del dolor debe ser resuelta. Por último, señalan que existe un largo debate alrededor del problema de los efectos adversos, si es que los mismos valen los beneficios del uso de anticonceptivos orales en el control de la dismenorrea

Los anticonceptivos orales son altamente efectivos si se administran de forma correcta tomando en cuenta las indicaciones, 
indica Montero (2011), quien extiende que esta forma de anticoncepción no afecta la actividad sexual y están constituidos por "un estrógeno (etinil estradiol) y por un progestágeno (levonorgestrel, norgestimato, gestodeno, desogestrel, clormadinona, ciproterona, drospirenona, dienogest, los últimos 4 con mayor efecto antiandrogénico)" (pág. 61).

Así mismo, estos medicamentos brindan beneficios no asociados con la prevención de embarazo, como el alivio del acné, reducción de quistes ováricos, prevención del cáncer endometrial y de ovario, así como la enfermedad fibroquistica benigna de la mama y la enfermedad pélvica inflamatoria. (Hernández, Quesada, Valdés, García \& Boza, 2007)

Sin embargo, una de las desventajas del uso de anticonceptivos orales son los posibles efectos secundarios que acompañan al uso de las dos hormonas que contienen los mismos. Los efectos secundarios relacionados a la alteración en los niveles de estrógeno pueden incluir vómitos, náuseas y dolor de cabeza, mientras que en el caso de los progestágenos el aumento del crecimiento del cabello y depresión. Cabe señalar que los niveles de estas hormonas han ido reduciendo sus dosis a medida que evolucionan los medicamentos lo que ayuda a disminuir los efectos adversos. (Wong et al., 2009)

Por otro lado, Hernández et al. (2007) explican que:

El bloqueo precoz del eje hipotálamo-hipofisario no tiene repercusiones negativas comprobadas en el organismo ni parece afectar el pico de masa ósea. Los anticonceptivos orales tampoco son responsables del aumento mantenido de peso, y el incremento del cáncer de mama detectado en mujeres que usaron preparados diferentes y de altas dosis de estrógeno en su adolescencia, no se ha demostrado con las formulaciones actuales.
Mientras que, Montero (2011) señala que con los preparados que se utilizan actualmente, los efectos adversos así como los riesgos relacionados al uso de anticonceptivos orales han sido reducidos y estos traen consigo beneficios como el control del flujo menstrual, infecciones pelvianas y disminución de dismenorrea.

En cuanto al uso de anticonceptivos orales en el tratamiento de la dismenorrea, estudios clínicos muestran que es efectivo debido a la inhibición de la ovulación y disminución de los niveles de prostaglandinas que producen estos medicamentos. En un estudio realizado por Gauthier en 1992, se descubrió que, de una muestra de 661 mujeres, casi dos tercios de ellas experimentaron dismenorrea, de las cuales solo el $12 \%$ siguió padeciéndola luego de ingerir anticonceptivos orales durante 12 meses. Mientras que, en otro estudio de 1991, conducido por Billy, esta vez con una muestra notablemente mayor de 100.000 mujeres quienes sufrieron episodios de dismenorrea, se dio a conocer que el $65 \%$ de ellas, quienes utilizaron anticonceptivos orales por primera vez sintieron alivio de los dolores producidos por la patología. Por lo tanto, existe evidencia que apunta a la popularidad de los anticonceptivos orales en el tratamiento efectivo de la dismenorrea. (Wong et al., 2009)

Amaya (2010) añade que:

Dada su acción sobre el folículo ovárico disminuyen la síntesis de progesterona, con lo cual se tiene un menor sustrato para formación de fosfolípidos, menor síntesis de ciclooxigenasa, menor concentración de prostaglandinas y menstruaciones mucho más escasas. En general se considera que se produce mejoría en el 90\% de las pacientes, siendo por tanto los anticonceptivos orales combinados de micro dosis la primera alternativa de anticoncepción en aquellas mujeres que presentan dismenorrea primaria y que desean a la vez realizar planificación familiar (pág. 297). 
Cabe señalar que, una de las ventajas de los anticonceptivos orales es su acceso libre y sin preescripcion, asi como los altos conocimientos que poseen sus usuarias en cuanto a su uso correcto, como lo determinó un estudio realizado por Ferrer, Murillo \& Machuca (2003) donde se encuestaron 100 mujeres que utilizaban frecuentemente estos medicamentos.

Asimismo, otros de los medicamentos también recomendados para el control de la dismenorrea son los antiinflamatorios no esteroides (AINES), a lo que Amaya (2010) explica que:

Tienen ventajas sobre los anticonceptivos orales combinados, ya que sólo necesitan ser administrados por dos o tres días al mes y al disminuir la hipercontractilidad miometrial, mejoran otras manifestaciones de la dismenorrea como vértigos, náuseas y vómitos. El aumento en la tasa de éxito con el uso de los AINES, ha llevado a que hoy día sean los medicamentos de primera línea y a que una larga lista de productos esté comercialmente disponible para el manejo de la dismenorrea primaria. Aquellos casos de falla en la respuesta a los AINES, son los que llevan a pensar que otros mediadores, como los leucotrienos, están presentes en la fisiopatología de la dismenorrea primaria (pág. 296).

Cabe destacar que uno de los beneficios que ofrece los anticonceptivos orales está relacionado al control maternal, siendo una opción viable para aquellas mujeres que deseen también prevenir el embarazo, aumentando el valor de este tratamiento.

Por otra parte, Jiménez et al. (2013) exponen que:

El consumo de anticonceptivos hormonales también puede explicar la diferencia encontrada entre la prevalencia en vida y la actual puesto que la cifra de estudiantes que los consumen es alta (61.5\%) y de este porcentaje más de una tercera parte $(34.5 \%)$ considera que la frecuencia o intensidad de la dismenorrea ha mejorado gracias a ellos. En este sentido debe tenerse en cuenta que la evidencia actual sugiere que ciertos regímenes basados en anticonceptivos orales favorecen la disminución de los síntomas de la dismenorrea. Se infiere que los anticonceptivos orales combinados evitan la ovulación y restringen el crecimiento endometrial, lo cual hace que disminuya la producción de prostaglandinas y el volumen catamenial y contribuye a mejorar el cuadro (pág. 25).

En lo que respecta a la eficacia que tiene los anticonceptivos orales en el tratamiento de la dismenorrea, Wong et al. (2009) analizan diversos estudios relacionados a este tópico. Uno de ellos, un estudio epidemiológico longitudinal sobre 500 mujeres suizas de 19 años de edad donde se comparó la ocurrencia y severidad de la dismenorrea en aquellas que ingerían anticonceptivos orales en contraste de las que no, donde luego de 5 años las primeras experimentaron una reducción en la prevalencia y dureza de esta patología mayor al del segundo grupo. A pesar de esto, los cambios fueron atribuidos en parte al paso del tiempo en lugar de la eficacia de los anticonceptivos orales.

En este orden de ideas, Amaya (2010) señala que si no hay mejoría luego de consumir los fármacos recomendados, es obligatorio primeramente revalorizar el caso para encontrar las posibles modificaciones ginecológicas o no ginecológicas, lo que determinaría la presencia de dismenorrea secundaria.

Por otra parte, Schulin \& Conejero (2011) exponen que:

Se recomienda hacer prueba de 3 meses, en los casos que no exista respuesta al dolor, se pueden utilizar ACO en forma continua por un período de 4 meses o realizar una laparoscopía. La dismenorrea recidivante y progresiva pese al uso de ACO, debe hacernos sospechar la existencia de patología orgánica particularmente endometriosis

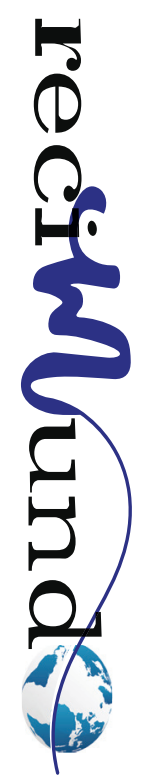


y realizarse una laparoscopía (pág. 47).

\section{Conclusiones}

Debido a que la dismenorrea es causada por un alto volumen del flujo menstrual, y debido a que los anticonceptivos orales ayudan a regular dicho flujo, estos pueden ser utilizados como tratamiento para esta problemática. Por otra parte, si el uso de estos medicamentos no ofrece una mejoría significativa, se debe considerar la presencia de dismenorrea secundaria, la cual puede estar originada por una patología que los anticonceptivos no pueden aliviar.

A lo que se concluye que la ingesta de anticonceptivos orales para reducir la producción de prostaglandinas constituye un tratamiento al cual su eficacia no ha sido totalmente comprobada. Por lo tanto, se debe considerar también el uso de antiinflamatorios no esteroides y en última instancia, si los síntomas no son reducidos, se debe realizar una exploración en busca de problemas pélvicos o patologías ginecológicas que puedan originar episodios de dismenorrea secundaria.

\section{Bibliografía}

Amaya, G. (2010). Dismenorrea. Ginecología y Obstetricia, 12. Recuperado el 5 de Febrero de 2020, de http://aprendeenlinea.udea.edu.co/revistas/ index.php/ginecologia_y_obstetricia/article/viewFile/17549/15142

Casey, F. (Septiembre de 2018). Anticonceptivos orales - Ginecología y obstetricia - Manual MSD versión para profesionales. Recuperado el 8 de Febrero de 2020, de Manual MSD versión para profesionales: https://www.msdmanuals.com/es/ professional/ginecolog\%C3\%ADa-y-obstetricia/ planificaci\%C3\%B3n-familiar/anticonceptivos-orales

Escalonilla, B., Rodríguez, Á., Marroyo, J., \& Lillo, R. (2010). Frecuencia y características de la dismenorrea en mujeres de la zona de salud de Torrijos (Toledo). Enfermería Clínica, 20(1), 32-35. doi:10.1016/j.enfcli.2009.09.001

Ferrer, I., Murillo, M., \& Machuca, M. (2003). Determinación del conocimiento y correcta utilización sobre anticonceptivos orales en farmacia comunitaria. Pharmacy Practice, 1(3), 136-138. Obtenido de https://www.redalyc.org/pdf/690/69010309.pdf

Hernández, J. C., Quesada, M. Y., Valdés, A. I., García, P. H., \& Boza, A. V. (2007). Anticoncepción en la adolescencia. Revista Cubana de Endocrinología, 18(1). Recuperado el 7 de Febrero de 2020, de http://scielo.sld.cu/scielo.php?script=sci_arttext\&pid=S1561-29532007000100006

Jiménez, G., González, J., Mesa, H., \& Capera, Y. (2013). Prevalencia, factores de riesgo y características clínicas de la dismenorrea en estudiantes de la Facultad de Enfermería de la Universidad Pontificia Bolivariana. Medicina Universidad Pontificia Bolivariana, 20-29. Recuperado el 6 de Febrero de 2020, de https://revistas.upb.edu.co/ index.php/Medicina/article/view/2123/1920

Montero, A. (2011). Anticoncepción en la adolescencia. Revista Médica Clínica CONDES, 22(1), 5967. Recuperado el 06 de Febrero de 2020

Muñoz, P., Camacho, J., Alvarado, O., \& Macías, M. (Julio de 2018). La dismenorrea como principal causante de periodos menstruales doloroso. Revista Científica de Investigación actualización del mundo de las Ciencias, 3(3), 769-788. doi:10.26820/reciamuc/3.(3).julio.2019.769-788

Oficina para la Salud de la Mujer. (24 de Abril de 2017). Métodos anticonceptivos | womenshealth. gov. Recuperado el 8 de Febrero de 2020, de Sitio web de womenshealth.gov: https://espanol.womenshealth.gov/a-z-topics/birth-control-methods

Ortiz, M., Pérez, N., Macías, A., Carrillo, L., Rangel, E., Fernández, E., \& Ponce, H. (2007). Utilización de fármacos para el tratamiento de dismenorrea primaria en estudiantes universitarias. Revista Mexicana de Ciencias Farmacéuticas, 38(4), 2429. Recuperado el 5 de Febrero de 2020

Sandoval, J., Madrid, A., Sandoval, C., \& Paz, P. (2013). Factores que condicionan la dismenorrea en adolescentes, según estrato socioeconómico. Revista Peruana de Ginecología y Obstetricia, 59(2). Recuperado el 06 de Febrero de 2020, de http://www.scielo.org.pe/scielo.php?pi$d=$ S2304-51322013000200003\&script=sci_arttext\&tlng =en

Schulin, C., \& Conejero, C. (2011). Trastornos menstruales y dismenorrea en la adolescencia. Revista Medica Clinica Condes, 22(1), 39-47. Recuperado el 5 de Febrero de 2020, de https://pdf.sciencedirectassets. com/312299/1-s2.0-S0716864011X70189/1s2.0-S0716864011703911/main.pdf

Wong, C., Farquhar, C., Roberts, H., \& Proctor, M. (7 de Octubre de 2009). Oral contraceptive 
pill for primary dysmenorrhoea. doi:https://doi. org/10.1002/14651858.CD002120.pub3

Yañez, N., Bautista, S., Ruiz, J., \& Ruiz, Á. (2010). Prevalencia y factores asociados a dismenorrea en estudiantes de ciencias de la salud. Revista Ciencia Salud, 8(3), 37-48. Recuperado el 7 de Febrero de 2020, de https://www.redalyc.org/ pdf/562/56220004002.pdf

\section{CITAR ESTE ARTICULO:}

Díaz Chávez, A., Jaramillo Moreno, J., Aguilar Cobo, A., \& Haro Perdomo, A. (2020). Uso de anticonceptivos orales en la dismenorrea. RECIMUNDO, 4(1 (Esp)), 155-163. doi:10.26820/recimundo/4.(1).esp.marzo.2020.155-163

\section{(c) $(1)(0$ BY NC SA}

RECONOCIMIENTO-NOCOMERCIAL-COMPARTIRIGUAL CC BY-NC-SA

ESTA LICENCIA PERMITE A OTROS ENTREMEZCLAR, AJUSTARY ESTA LICENCIA PERMITE A OTROS ENTREMEZCLAR, AJUSTARY
CONSTRUIR A PARTIR DE SU OBRA CON FINES NO COMERCIALES, SIEMPRE Y CUANDO LE RECONOZCAN LA AUTORÍA Y SUS NUEVAS CREACIONES ESTÉN BAJO UNA LICENCIA CON LOS MISMOS TÉRMINOS. 